\title{
AMBIGUITY AND COMMUNICATION
}

\author{
JURAJ HROMKOVIC ${ }^{1}$ AND GEORG SCHNITGER ${ }^{2}$ \\ ${ }^{1}$ Department of Computer Science, ETH Zürich, \\ ETH Zentrum, CH-8022 Zürich, Switzerland \\ E-mail address: juraj.hromkovic@inf.ethz.ch \\ ${ }^{2}$ Institut für Informatik, Goethe Universität, \\ Robert Mayer-Strasse 11-15, D-6054 Frankfurt a. M., Germany \\ E-mail address: georg@thi.informatik.uni-frankfurt.de
}

\begin{abstract}
The ambiguity of a nondeterministic finite automaton (NFA) $N$ for input size $n$ is the maximal number of accepting computations of $N$ for an input of size $n$. For all $k, r \in \mathbb{N}$ we construct languages $L_{r, k}$ which can be recognized by NFA's with size $k \cdot \operatorname{poly}(r)$ and ambiguity $O\left(n^{k}\right)$, but $L_{r, k}$ has only NFA's with exponential size, if ambiguity $o\left(n^{k}\right)$ is required. In particular, a hierarchy for polynomial ambiguity is obtained, solving a long standing open problem (Ravikumar and Ibarra, 1989, Leung, 1998).
\end{abstract}

\section{Introduction}

The ambiguity of an NFA $N$ measures the degree of nondeterminism employed by $N$ as a function of the input size: let $\operatorname{ambig}_{N}(x)$ be the number of accepting computations of $N$ on input $x$ and define

$$
\operatorname{ambig}_{N}(n)=\max \left\{\operatorname{ambig}_{N}(x): x \in \Sigma^{n}\right\}
$$

to be the ambiguity of $N$. There are related complexity measures such as the advice and the leaf complexity of $N$. To describe their definition let $T_{N}(x)$ be the computation tree of $N$ on input $x$. Then advice ${ }_{N}(x)$ is the maximum, over all paths in $T_{N}(x)$ from the root to a leaf, of the number of nodes with at least two children and

$$
\operatorname{advice}_{N}(n)=\max \left\{\operatorname{advice}_{N}(x): x \in \Sigma^{n}\right\}
$$

is the advice complexity of $N$. The leaf complexity of $N$ determines the maximal number of computations for inputs of length $n$. Thus, if leaf ${ }_{N}(x)$ is the number of leaves of $T_{N}(x)$, then

$$
\operatorname{leaf}_{N}(n)=\max \left\{\operatorname{leaf}_{N}(x): x \in \Sigma^{n}\right\} .
$$

For a minimal NFA $N$ these measures are related as follows [2]

$$
\operatorname{advice}_{N}(n), \operatorname{ambig}_{N}(n) \leq \operatorname{leaf}_{N}(n)=O\left(\operatorname{advice}_{N}(n) \cdot \operatorname{ambig}_{N}(n)\right)
$$

Key words and phrases: Nondeterministic finite automata, ambiguity, communication complexity.

Supported by SNF-grant 200020-120073 and DFG-grant SCHN 503/4-1. Part of the work was done while the second author was visiting the ETH Zürich.

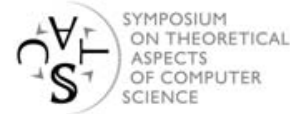


and, since $\operatorname{advice}_{N}(n)$ is at most linear, leaf complexity and ambiguity are polynomially related, provided both are at least linear. Since leaf complexity is either bounded by a constant or at least linear but polynomially bounded, or otherwise exponential in the input length, we obtain that ambiguity is either bounded by a constant or bounded by a polynomial or at least exponential [2].

Advice and leaf complexity are rather coarse measures, since advice and leaf complexity of an unambiguous NFA may be linear. Ambiguity on the other hand also influences the tractability of algorithmic questions. For instance, for any fixed $k \in \mathbb{N}$ it can be determined efficiently whether two NFA's of ambiguity at most $k$ are equivalent, resp. whether the ambiguity of a given NFA is at most $k$ [7].

How large is the decrease in conciseness, i.e., the increase in the number of states, if ambiguity is restricted? To study this question, four classes of NFA's, namely UNA (unambiguous nondeterministic automata), FNA (finitely ambiguous NFA), PNA (polynomially ambiguous NFA) and ENA (exponentially ambiguous NFA) are introduced in [6]. The classification into FNA's, PNA's or ENA's can be performed efficiently [8].

Remember that the ambiguity of an NFA $N$ is either at least exponential or at most polynomial and hence an NFA is either a PNA or an ENA. Leung [4] shows that there are ENA's $N_{n}$ with $n$ states such that any equivalent PNA has at least $2^{n}-1$ states. Hence ENA's can be far more succinct than PNA's. Subsequently a similar result, applicable to a larger class of languages, was shown in [2] by using methods of communication complexity. In particular, the conciseness problem for PNA's can be reduced to the following communication result for the iterated language of non-disjointness. Let $\Sigma_{r}$ be the alphabet of all subsets of $\left\{1, \ldots, r^{32}\right\}$ of size $r$ and set

$$
L_{r}=\left\{x y \mid x, y \in \Sigma_{r} \text { and } x \cap y \neq \emptyset\right\} .
$$

Thus $\left(L_{r}\right)^{t}$ consists of all strings $x_{1} y_{1} \cdots x_{t} y_{t}$ where all pairs $x_{i} y_{i}$ correspond to overlapping subsets. We assume the standard communication model with two players, Alice and Bob, where Alice receives $x_{1} \cdots x_{t}$ and Bob receives $y_{1} \cdots y_{t}$. (Observe that $\left(L_{r}\right)^{t}$ has small NFA's with poly $(r+t)$ states.)

Fact 1.1. ([3], pages 51-53). Let $r, t \in \mathbb{N}$ be arbitrary. If a deterministic protocol $D$ accepts only strings from $\left(L_{r}\right)^{t}$ and if at most $2^{\alpha \cdot r \cdot t}$ messages are exchanged, then $D$ accepts at most $\left|\left(L_{r}\right)^{t}\right| / 2^{\alpha \cdot t}$ strings from $\left(L_{r}\right)^{t}$. ( $\alpha$ is a sufficiently small constant).

Of particular interest are FNA's, for instance since their equivalence problem is efficiently solvable. However a separation of FNA's and PNA's has remained open for almost twenty years $[4,6]$. We are able to show such a separation and even prove a hierarchy result for polynomial ambiguity. To describe our result we introduce the languages used in the separation. For a language $L$ of strings of identical length define

$$
\exists_{k}(L)=\left\{w_{1} w_{2} \cdots w_{m} \mid m \in \mathbb{N} \text { and } w_{i} \in L \text { for at least } k \text { different positions }\right\} .
$$

Thus the input is partitioned into blocks of identical length and an input is accepted iff at least $k$ blocks belong to the finite set $L$. Now assume that $L$ can be recognized by a small NFA $N$. Since $L$ is a finite set, we can recognize $\exists_{k}(L)$ by an NFA with ambiguity $O\left(n^{k}\right)$, if we increase the size of $N$ by at most the factor $k$.

How should the languages $L$ look like? In a first attempt set $L=\left\{u v \mid u, v \in\{0,1\}^{r}, u \neq\right.$ $v\}$ as the language of inequality between $r$-bit strings. Then $L$ is recognizable by an NFA with poly $(r)$ states and (bounded) ambiguity $r$. But $\exists_{1}(L)$ is also recognizable with poly $(r)$ 
states and ambiguity $r$ : guess a position $i \in\{1, \ldots, r\}$ and accept $u^{1} v^{1} \cdots u^{m} v^{m}$ if $u_{i}^{j} \neq v_{i}^{j}$ for some $1 \leq j \leq r$.

What went wrong? Few advice bits suffice and these advice bits can be remembered. In our second (and successful) attempt we therefore set $L=\left(L_{r}\right)^{t}$, where we work with the iterated language of non-disjointness from Fact 1.1. This construction has two advantages. Firstly, $L$ has a small NFA. Secondly, at least intuitively, the number of guesses required for $L$ increases exponentially with $t$ and hence a small NFA's for $\exists_{1}\left(L_{r}\right)$ cannot remember sequences of $t$ guesses. Our main result verifies this intuition.

Theorem 1.2. Let $r \in \mathbb{N}$ be arbitrary. Set $t=r^{1 / 3}$ and $L=\left(L_{r}\right)^{t}$. Any NFA for $\exists_{k}(L)$ with ambiguity $o\left(n^{k}\right)$ has at least $2^{\Omega\left(\left(r / k^{2}\right)^{1 / 3}\right)}$ states. However, $\exists_{k}(L)$ can be recognized by an NFA with ambiguity $O\left(n^{k}\right)$ and size $k \cdot \operatorname{poly}(r)$.

Observe that we have obtained the claimed separation of FNA's and PNA's for $k=1$, but Theorem 1.2 also establishes a hierarchy of polynomial ambiguity.

\section{A Proof Sketch}

We start by proving Theorem 1.2 for $k=1$. Let $L=\left(L_{r}\right)^{t}$ and assume that the NFA $N$ recognizes $\exists_{1}(L)$ with sublinear ambiguity. Observe that all strings in $L$ have length $2 t$ and hence strings in $\exists_{1}(L)$ have blocks of identical length $2 t$. We set $K=\Sigma_{r}^{2 t}$, where $\Sigma_{r}$ is the alphabet of $L_{r}$. Finally set

$$
\exists_{=0}(L)=\left\{w_{1} w_{2} \cdots w_{m}: m \in \mathbb{N} \text { and } w_{i} \in K \backslash L \text { for all } i\right\} .
$$

Thus, as in the definition of $\exists_{1}(L)$, the input is partitioned into blocks and an input is accepted iff no block belongs to the finite set $L$. The computationally hardest task for the NFA $N$ is to separate $\exists_{=0}(L)$ from $\exists_{1}(L)$.

The critical part of the argument is to exploit the limitation of sublinear ambiguity. Let $Q$ be the set of states of $N$. In Section 3 we construct states $p_{0}, p_{1} \in Q$ such that at least $|L| /|Q|^{2}$ strings in $L$ have a computation starting in $p_{0}$ and ending in $p_{1}$. Moreover we show in Lemma 3.3 that for any string $z^{\prime} \in K \backslash L$ there is a string $u \in \exists_{=0}(L)$ such that strings $S\left(z^{\prime}\right)$ with period $z^{\prime} u$ can be "stored" in a "launching cycle" before reaching $p_{0}$ and in a "storage cycle" after leaving $p_{1}$. The launching cycle has the form $r \stackrel{\left(z^{\prime} u\right)^{a}}{\rightarrow} r$ and allows to reach $p_{0}$ via a computation $r \stackrel{\left(z^{\prime} u\right)^{a_{1}}}{\longrightarrow} p_{0}$; analogously the storage cycle is built from computations $p_{1} \stackrel{\left(u z^{\prime}\right)^{a_{2}}}{\rightarrow} s$ and $s \stackrel{\left(u z^{\prime}\right)^{a}}{\rightarrow} s$. So far the launching cycle is harmless, since it delivers strings in $\exists_{=0}(L)$ to state $p_{0}$, but these strings cannot use computations from $p_{0}$ to $p_{1}$ which may be reserved for strings in $L$. However, if a single occurrence of $z^{\prime}$ within $S\left(z^{\prime}\right)$ is replaced by an impostor string $z \in L$ and if the launching cycle does not detect the replacement, then $N$ is forced into linear ambiguity, provided the impostor $z$ can also hide at a matching position within the storage cycle (see Lemma 3.4).

Thus the NFA $N$ has to solve the "detection problem", namely it has to detect whether an impostor $z \in L$ has replaced an occurrence of $z^{\prime} \in K \backslash L$ in both cycles. The detection problem is set up in such a way that

- at least $|L| /|Q|^{2}$ strings from $L$ are accepted, namely those strings $z \in L$ with a computation $p_{0} \stackrel{z}{\rightarrow} p_{1}$, and 
- all strings $z$ which for some $z^{\prime} \in K \backslash L$ survive in matching positions within both cycles are rejected. In particular, all strings in $K \backslash L$ are rejected, since a string $z \in K \backslash L$ is its own impostor.

Observe that no string $z$ is simultaneously accepted as well as rejected, since all impostors have to be detected. $N$ may try to solve the detection problem unconventionally for instance by allowing a potential impostor $z$ to survive undetected within the launching and storage cycle, but not allowing $z$ to survive in matching positions within both cycles. Also $N$ does not have to solve the detection problem completely, since it can tolerate an impostor $z$ without a computation $p_{0} \stackrel{z}{\rightarrow} p_{1}$.

We then simulate $N$ in Section 4 by a nondeterministic communication protocol which rejects all strings in $K \backslash L$, accepts at least $|L| /|Q|^{2}$ strings in $L$ and does not simultaneously accept and reject a string in $K \backslash L$ (see Lemma 4.1). Thus we have reduced the problem of avoiding linear ambiguity for NFA's recognizing $\exists_{1}(L)$ to a communication problem in which a rather small minority of strings in $L$ has to be separated from all of $K \backslash L$. We show in Lemma 5.1 how to transform such a nondeterministic protocol into a deterministic protocol by increasing the number of messages only subexponentially. We are left with a deterministic protocol which rejects all strings in $K \backslash L$ and accepts at least $|L| /|Q|^{2}$ strings in $L$. Finally the argument concludes with an application of Fact 1.1. Thus, as in the case of exponential ambiguity, we again have reduced the conciseness problem to an investigation of deterministic protocols which recognize a "small, but significant chunk" of a given product language.

The general case of ambiguity $O\left(n^{k}\right)$ is tackled in Section 6. Showing the existence of launching and storage cycles has now become a more complex problem. Previously it was sufficient that the periodic string $S(z)$ was "living" in the one launching and the one storage cycle. Now we have to work with a vector $p_{0}, p_{1}, \ldots, p_{2 k-2}, p_{2 k-1}$ of states and have to move $S(z)$ to $p_{0}$ and all the way from $p_{2 i+1}$ to $p_{2(i+1)}$ for all $i=0, \ldots k-2$ and finally from $p_{2 k-1}$ to an accepting state.

\section{From Automata to Communication}

We begin by utilizing the special structure of the languages $\exists_{1}(L)$.

Definition 3.1. Let $N$ be an NFA for $\exists_{1}(L)$ with initial state $q_{0}$. Let $p$ be an arbitrary state of $N$.

(a) We say that a string $v \in \exists_{=0}(L)$ reaches state $p$ iff there is a string $u \in \exists_{=0}(L)$ and a computation for $u \cdot v$ which starts in $q_{0}$ and ends in $p$. Moreover state $p$ accepts $v \in \exists_{=0}(L)$ iff there is a string $w \in \exists_{=0}(L)$ and an accepting computation for $v \cdot w$ starting in $p$.

(b) A pair $\left(p_{0}, p_{1}\right)$ of states of $N$ is critical for the pair $\left(\xi_{0}, \xi_{1}\right) \in \exists_{=0}(L) \times \exists_{=0}(L)$ iff all strings in $\exists_{=0}(L) \cdot \xi_{0}$ reach $p_{0}$ and all strings in $\xi_{1} \cdot \exists_{=0}(L)$ are accepted by $p_{1}$.

Our next goal is to construct a pair $\left(\xi_{0}, \xi_{1}\right) \in \exists_{=0}(L) \times \exists_{=0}(L)$ such that for all strings $u \xi_{0} z \xi_{1} w \in \exists_{=0}(L) \cdot\left(\xi_{0} \cdot L \cdot \xi_{1}\right) \cdot \exists_{=0}(L)$ acceptance is "decided" by critical pairs. In particular we construct $\left(\xi_{0}, \xi_{1}\right)$ such that there are accepting computations of the form $q_{0} \stackrel{u \xi_{0}}{\rightarrow} p_{0} \stackrel{z}{\rightarrow}$ $p_{1} \stackrel{\xi_{1} w}{\longrightarrow} q_{f}$ for a final state $q_{f}$ and a critical pair $\left(p_{0}, p_{1}\right)$ for $\left(\xi_{0}, \xi_{1}\right)$. The crucial advantage of a critical pair is that all strings in $\exists_{0}(L) \cdot \xi_{0}$ reach $p_{0}$ and all strings in $\xi_{1} \cdot \exists_{0}(L)$ are accepted 
by $p_{1}$; in particular, there is no transition $p_{0} \stackrel{z}{\rightarrow} p_{1}$ for a string $z \in \exists_{0}(L)$ and acceptance is indeed decided by $\left(p_{0}, p_{1}\right)$.

Lemma 3.2. Let $N$ be an $N F A$ for $\exists_{1}(L)$. Then there are strings $\xi_{0}, \xi_{1} \in \exists_{=0}(L)$ such that

$$
\bigcup_{\left(p_{0}, p_{1}\right) \text { is critical for }\left(\xi_{0}, \xi_{1}\right)}\left\{z \in L \mid p_{0} \stackrel{z}{\rightarrow} p_{1}\right\}=L .
$$

Proof. We process the states of $N$ in two phases. In the first phase we construct a string $\xi_{0} \in \exists_{=0}(L)$ such that each state $p$ is either alive for $\xi_{0}$ (i.e., all strings in $\exists_{=0}(L) \cdot \xi_{0}$ reach $p$ ) or dead for $\xi_{0}$ (i.e., no string in $\exists_{=0}(L) \cdot \xi_{0}$ reaches $p$ ). The construction process proceeds iteratively by processing all states $p$ of $N$ in an arbitrary order. We begin by setting $\xi_{0}=\epsilon$. When processing state $p$ we differentiate two cases.

Case 1: All strings in $\exists_{=0}(L) \cdot \xi_{0}$ reach $p$. We do not modify $\xi_{0}$. Observe that $p$ is alive for $\xi_{0}$ and stays alive for any string in $\exists_{=0}(L)$ with suffix $\xi_{0}$.

Case 2: There is a string $\xi \in \exists_{=0}(L)$ such that $\xi \cdot \xi_{0}$ does not reach $p$. The string $\xi \cdot \xi_{0}$ does not reach $p$ and hence no string in $\exists_{=0}(L) \cdot \xi \cdot \xi_{0}$ has a computation beginning in the starting state $q_{0}$ and ending in $p$. We replace $\xi_{0}$ by $\xi \cdot \xi_{0}$ and $p$ is dead for $\xi_{0}$, but also dead for any string in $\exists_{=0}(L)$ with suffix $\xi_{0}$. Also observe that any already processed state $q$ stays alive, resp. remains dead.

In the second phase we proceed completely analogously, but now construct a string $\xi_{1} \in \exists_{=0}(L)$ such that each state $p$ is either alive for $\xi_{1}$ (i.e., $p$ accepts all strings in $\left.\xi_{1} \cdot \exists_{=0}(L)\right)$ or dead for $\xi_{1}$ (i.e., $p$ does not accept any string in $\xi_{1} \cdot \exists_{=0}(L)$ ).

Now consider any string $s=\xi_{0} z \xi_{1}$ in $M=\xi_{0} \cdot L \cdot \xi_{1}$. Observe that $M$ is a subset of $\exists_{1}(L)$. However $\xi_{0}$ cannot reach a dead state for $\xi_{0}$ and $\xi_{1}$ cannot be accepted by a dead state for $\xi_{1}$. Thus any accepting computation for $s$ has to utilize a transition $p_{0} \stackrel{z}{\rightarrow} p_{1}$ between alive states $p_{0}$ for $\xi_{0}$ and $p_{1}$ for $\xi_{1}$. But any pair $\left(p_{0}, p_{1}\right)$ of alive states is a critical pair and we are done.

From now on we fix a pair $\left(\xi_{0}, \xi_{1}\right) \in \exists_{=0}(L) \times \exists_{=0}(L)$ for which Lemma 3.2 holds. Let $\left(p_{0}, p_{1}\right)$ be an arbitrary critical pair for $\left(\xi_{0}, \xi_{1}\right)$. We now utilize that all strings in $\exists_{=0}(L) \cdot \xi_{0}$ reach $p_{0}$ and all strings in $\xi_{1} \cdot \exists_{=0}(L)$ are accepted by $p_{1}$.

Lemma 3.3. For all strings $z \in K \backslash L$ there are states $r, s$, integers $a \geq 1, a_{1}, a_{2}$ (with $\left.a_{1}+a_{2} \leq a\right)$ and a string $u \in \exists_{=0}(L)$ as well as computations

$$
\begin{aligned}
& r \stackrel{(z u)^{a}}{\longrightarrow} r \stackrel{(z u)^{a_{1}}}{\rightarrow} p_{0} \text { and } \\
& p_{1} \stackrel{(u z)^{a_{2}}}{\rightarrow} s \stackrel{(u z)^{a}}{\rightarrow} s .
\end{aligned}
$$

Proof. We consider all strings of the form

$$
\alpha(z)=\left(z \xi_{1} \xi_{0}\right)^{|Q|} \text { and } \beta(z)=\left(\xi_{1} \xi_{0} z\right)^{|Q|} \text {. }
$$

The string $\alpha(z)$ has suffix $\xi_{0}$ and hence $\alpha(z)$ reaches $p_{0}$. As a consequence there is $\xi \in \exists_{=0}(L)$ and a computation $C$ for $\xi \cdot \alpha(z)$ which begins in the initial state $q_{0}$ and reaches $p_{0}$. After reading $\xi$, computation $C$ processes $\alpha(z)$ and produces a sequence of $|Q|+1$ states, where we list all states before reading a copy of $z \xi_{1} \xi_{0}$, resp. after reading the last copy. A state $r$ of $N$ appears twice in this sequence and we obtain a transition of the form $r \stackrel{\left(z \xi_{1} \xi_{0}\right)^{a}}{\rightarrow} r$ for $a \geq 1$. Finally $C$, starting in $r$, reaches $p_{0}$ after reading the remaining $a_{1}$ copies.

To establish (3.1), we set $u=\xi_{1} \xi_{0}$ and obtain transitions $r \stackrel{(z u)^{a}}{\longrightarrow} r$ and $r \stackrel{(z u)^{a}{ }^{\prime}}{\longrightarrow} p_{0}$. Thus (3.1) follows. Part (3.2) is established by a similar argument, but now applied to $\beta(z)$. This 
time we get transitions $p_{1} \stackrel{(u z)^{a_{2}}}{\rightarrow} s$ and $s \stackrel{(u z)^{b}}{\rightarrow} s$. But then $r \stackrel{(z u)^{m a}}{\rightarrow} r$ as well as $s \stackrel{(u z)^{m^{\prime} b}}{\rightarrow} s$ are transitions for any multiples $m, m^{\prime} \geq 1$ and the claim follows, if we replace both $a$ and $b$ by $a b\left(a_{1}+a_{2}\right) \geq a_{1}+a_{2}$.

Let $\left(p_{0}, p_{1}\right)$ be a critical pair for $\left(\xi_{0}, \xi_{1}\right)$. We now introduce the detection problem for $\left(p_{0}, p_{1}\right)$ in which strings in $L$ have to be "weakly" separates from strings in $K \backslash L$. It turns out that any NFA $N$ for $\exists_{1}(L)$ solves the detection problems for all critical pairs, provided $N$ has ambiguity $o(n)$. Since we show later that $N$ can be efficiently simulated by a communication protocol -with communication resources related to the number of statesand that the detection problem is hard for communication complexity, $N$ must have many states. The detection problem of $\left(p_{0}, p_{1}\right)$ has the following form:

(a) Accept a string $z \in K$ iff there is a computation $p_{0} \stackrel{z}{\rightarrow} p_{1}$ of $N$. Remember that for no $z \in K \backslash L$ there is a computation

$$
q_{0} \stackrel{\xi \xi_{0}}{\rightarrow} p_{0} \stackrel{z}{\rightarrow} p_{1} \stackrel{\xi_{1} \xi^{\prime}}{\longrightarrow} q_{f}
$$

with the initial state $q_{0}$, a final state $q_{f}$ and strings $\xi, \xi^{\prime}, \xi_{0}, \xi_{1} \in \exists_{=0}(L)$. Hence no string $z \in K \backslash L$ is accepted.

(b) Reject a string $z \in K$ iff there are states $r, r^{\prime}, r^{\prime \prime}, s, s^{\prime}, s^{\prime \prime}$, integers $a \geq 1, a_{1}, a_{2}$ (with $\left.a_{1}+a_{2} \leq a\right)$ and strings $u \in \exists_{=0}(L), z^{\prime} \in K \backslash L$ with computations

$$
\begin{aligned}
& r \stackrel{\left(z^{\prime} u\right)^{a_{1}}}{\longrightarrow} r^{\prime} \stackrel{z u}{\longrightarrow} r^{\prime \prime} \stackrel{\left(z^{\prime} u\right)^{a-a_{1}-1}}{\rightarrow} r \stackrel{\left(z^{\prime} u\right)^{a_{1}}}{\longrightarrow} p_{0} \text { and } \\
& p_{1} \stackrel{\left(u z^{\prime}\right)^{a_{2}}}{\rightarrow} s \stackrel{\left(u z^{\prime}\right)^{a-a_{2}-1} \rightarrow}{\rightarrow} s^{\prime} \stackrel{u z}{\rightarrow} s^{\prime \prime} \stackrel{\left(u z^{\prime}\right)^{a_{2}}}{\longrightarrow} s .
\end{aligned}
$$

(The computations (3.3) and (3.4) will be used later to define a launching and storage cycle respectively. It turns out that $z$ is placed within matching positions of the $z^{\prime} u$ - and $u z^{\prime}$-cycle and hence $z$ plays the role of an impostor of $z^{\prime}$.)

(c) $z \in K$ is left undecided iff $z$ is neither accepted nor rejected.

To explain the purpose of these transitions consider the string

$$
S_{1}=\left[z u \cdot\left(z^{\prime} u\right)^{a-1}\right] \cdot\left[z u \cdot\left(z^{\prime} u\right)^{a-1}\right] .
$$

If we process the first half $z u \cdot\left(z^{\prime} u\right)^{a-1}$ of $S_{1}$ starting in state $r^{\prime}$, then there is a computation $C_{0}$ of the form

$$
r^{\prime} \stackrel{z u}{\rightarrow} r^{\prime \prime} \stackrel{\left(z^{\prime} u\right)^{a-a_{1}-1}}{\rightarrow} r \stackrel{\left(z^{\prime} u\right)^{a_{1}}}{\longrightarrow} r^{\prime}
$$

as well as a computation $C_{1}$ from $r^{\prime}$ to $p_{0}$ according to (3.3). When reading the second half of $S_{1}$, computation $C_{0}$ splits into a computation $C_{00}$ which goes full circle reaching state $r^{\prime}$ again and a computation $C_{01}$ which reaches $p_{0}$ after completely reading $S_{1}$. Now assume that there is a transition $p_{0} \stackrel{z}{\rightarrow} p_{1}$. Computation $C_{1}$ has reached $p_{0}$ after reading the first half of $S_{1}$ and now reads the second half $z u \cdot\left(z^{\prime} u\right)^{a-1}=z \cdot\left(u z^{\prime}\right)^{a-1} \cdot u$ of $S_{1}$. It travels from $p_{0}$ to $p_{1}$ and subsequently reaches state $s^{\prime \prime}$, if additionally the string $z$ is read. We have been successful

(1) in "storing" a mother computation via computation $C_{00}$ in state $r^{\prime}$,

(2) preparing for a new "launch" in state $p_{0}$ via computation $C_{01}$ and

(3) "storing" offspring computations in state $s$ " via computation $C_{1}$.

We utilize properties (1)-(3) by defining a sequence $\left(S_{m} \mid m \geq 1\right)$ with many computations, namely we set

$$
S_{m+1}=S_{m} \cdot\left[z u \cdot\left(z^{\prime} u\right)^{a-1}\right]=S_{m} \cdot\left[z \cdot\left(u z^{\prime}\right)^{a-1} \cdot u\right]
$$


Assume inductively that there are computations for $S_{m}$ which have reached the states $r^{\prime}$ and $p_{0}$ respectively and a computation for $S_{m} \cdot z$ which has reached $s^{\prime \prime}$. After reading the suffix $z u \cdot\left(z^{\prime} u\right)^{a-1}$ of $S_{m+1}$, the computation starting in $r^{\prime}$ has split into a computation reaching $r^{\prime}$ again and a computation reaching $p_{0}$, whereas the freshly launched computation reaches $s^{\prime \prime}$ from $p_{0}$ after reading $S_{m+1} \cdot z$. Observe that all previously launched computations go full circle after reading $\left(u z^{\prime}\right)^{a-1} \cdot u z$ and again have reached state $s^{\prime \prime}$. As a consequence, there are $m$ distinct computations for $S_{m} z$ all reaching state $s^{\prime \prime}$ at the same time.

We say that $N$ has no redundant states, if each state is part of some accepting computation of $N$. Which strings are rejected and which strings are accepted?

Lemma 3.4. Let $N$ be an $N F A$ recognizing $\exists_{1}(L)$ without redundant states. Also assume that $N$ has ambiguity o $(n)$.

(a) Consider the detection problem of an arbitrary critical pair $\left(p_{0}, p_{1}\right)$. Then all strings in $K \backslash L$ are rejected and no string in $K$ is simultaneously accepted and rejected.

(b) Each string in $L$ is accepted in the detection problem of some critical pair.

Proof. (a) We observe first that every string $z \in K \backslash L$ is rejected. Why? We may choose $z^{\prime}=z$ and the transitions required in (3.3) and (3.4) exist as a consequence of Lemma 3.3: the states $r^{\prime}, r^{\prime \prime}$ and $s^{\prime}, s^{\prime \prime}$ belong to the $r$-cycle and the $s$-cycle respectively.

Now assume that there is a string $z \in K$ which is accepted and rejected. Since $z$ is accepted, there is a computation $p_{0} \stackrel{z}{\rightarrow} p_{1}$. Also, since $z$ is rejected, there are computations of the form (3.3) and (3.4). Thus we may construct the strings $S_{m} z$ for every $m$ and obtain $m$ distinct computations which, starting from state $r^{\prime}$, reach state $s^{\prime \prime}$ at the same time. But $N$ does not have redundant states and each state, and in particular state $r^{\prime}$, is reachable from the initial state. Also each state, and in particular state $s^{\prime \prime}$, can reach an accepting state. Thus there are strings $\xi_{0}, \xi_{1}$ such that $\xi_{0} \cdot S_{m} z \cdot \xi_{1}$ has $m$ accepting computations. But $S_{m} z$ is a string with length linear in $m$ and hence $N$ has at least linear ambiguity.

(b) follows from part (a), if we apply Lemma 3.2.

\section{The Communication Problem}

We show that the detection problem has an efficient communication protocol, provided a small NFA $N$ with ambiguity $o(n)$ recognizes $\exists_{1}(L)$. Remember that $L=\left(L_{r}\right)^{t}$ and $K=\Sigma_{r}^{2 t}$. We work with the conventional two-party communication model consisting of two players Alice and Bob. If $x_{1} y_{1} \cdots x_{t} y_{t}$ is the input of $N$, then Alice receives $x_{1} \cdots x_{t}$ and Bob receives $y_{1} \cdots y_{t}$ as their respective inputs. Alice and Bob communicate nondeterministically with computations either being accepting, rejecting or undecided. We say that an input is accepted if at least one computation is accepting, rejecting if at least one computation is rejecting and undecided if all computations are undecided. (Thus undecided computations play the role of rejecting computations for conventional nondeterminism.) Observe that we allow to simultaneously accept and reject an input.

Now assume that the NFA $N$ recognizes $\exists_{1}(L)$. Let $q, q^{*}$ be two states of $N$ and let $z \in K$ be an input string. Our first goal is to determine whether $N$ has a computation for $z$ starting in $q$ and ending in $q^{*}$. Set $q_{0}=q$. Beginning with $i=1$, Alice simulates $N$ for input $x_{i}$ by starting in state $q_{i-1}$ and sends state $q_{i}^{\prime}$, if $q_{i}^{\prime}$ is reached. Bob simulates $N$ for input $y_{i}$ by starting in state $q_{i}^{\prime}$ and sends state $q_{i}$, if $q_{i}$ is reached. In the last round Bob accepts if additionally $q_{s}=q^{*}$ holds and otherwise outputs "undecided". Obviously the 
simulating protocol exchanges at most $|Q|^{2 t}$ messages. It has an accepting computation iff $N$ has a computation $q \stackrel{z}{\rightarrow} q^{*}$ and otherwise leaves the input undecided.

We say that a protocol solves the detection problem of $\left(p_{0}, p_{1}\right)$ if the protocol labels each input as accepted, rejected or undecided as prescribed by the detection problem.

Lemma 4.1. Assume that $N$ recognizes $\exists_{1}(L)$ and that $N$ has ambiguity o $(n)$. Let $\left(p_{0}, p_{1}\right)$ be a critical pair for $\left(\xi_{0}, \xi_{1}\right)$. Then there is a nondeterministic protocol $P$ which solves the detection problem of $\left(p_{0}, p_{1}\right)$ with $|Q|^{O(t)}$ messages.

Proof. We begin by describing the protocol $P$. In its first attempt $P$ tries to accept its input $z \in K$ by simulating the automaton $N$ when reading $z$ starting in state $p_{0} . P$ accepts $z$ iff state $p_{1}$ is reached and otherwise leaves $z$ undecided.

In its second attempt $P$ tries to reject $z$. Alice guesses states $r, r^{\prime}, r^{\prime \prime}, s, s^{\prime}, s^{\prime \prime}$ as well as strings $z^{\prime} \in K \backslash L, u \in \exists_{=0}(L)$ and integers $a_{1}, a_{2}, a$ (with $a_{1}+a_{2} \leq a$ ). Then Alice verifies the following transitions without communication, namely

$$
\begin{aligned}
& -r \stackrel{\left(z^{\prime} u\right)^{a_{1}}}{\longrightarrow} r^{\prime} \text { as well as } r^{\prime \prime} \stackrel{\left(z^{\prime} u\right)^{a-a_{1}-1}}{\rightarrow} r \stackrel{\left(z^{\prime} u\right)^{a_{1}}}{\longrightarrow} p_{0} \text { and } \\
& \text { - } p_{1} \stackrel{\left(u z^{\prime}\right)^{a_{2}}}{\longrightarrow} s \stackrel{\left(u z^{\prime}\right)^{a-a_{2}-1}}{\rightarrow} s^{\prime} \text { as well as } s^{\prime \prime} \stackrel{\left(u z^{\prime}\right)^{a_{2}}}{\longrightarrow} s .
\end{aligned}
$$

In order to check the remaining transition $r^{\prime} \stackrel{z u}{\rightarrow} r^{\prime \prime}$ and $s^{\prime} \stackrel{u z}{\rightarrow} s^{\prime \prime}$, Alice guesses additional states $\rho, \sigma$ and verifies the transitions $\rho \stackrel{u}{\rightarrow} r^{\prime \prime}$ and $s^{\prime} \stackrel{u}{\rightarrow} \sigma$ by herself. Subsequently Alice communicates the states $r^{\prime}, \rho$ as well as $\sigma, s^{\prime \prime}$ and both Alice and Bob simulate the automaton $N$ on input $z$ for starting states $r^{\prime}$ and $\sigma$. Bob rejects iff the transitions $r^{\prime} \stackrel{z}{\rightarrow} \rho$ and $\sigma \stackrel{z}{\rightarrow} s^{\prime \prime}$ have been verified and otherwise labels $z$ as undecided. Observe that $P$ exchanges at most $|Q|^{O(t)}$ messages, since $P$ uses messages only when simulating $N$ on the string $z \in K$.

\section{From Nondeterminism to Determinism}

In Lemma 4.1 we have solved the detection problem of a critical pair by a nondeterministic protocol $P$ with only $|Q|^{O(t)}$ messages. However the detection problem separates $L$ from its complement $K \backslash L$ only weakly, since the majority of strings from $L$ are either rejected or left undecided. We begin our analysis by transforming the nondeterministic protocol $P$ into a deterministic protocol $D$. We avoid an exponential blowup in the number of messages by observing the structural limitations of $P$. In particular, $P$ accepts a subset $L_{\text {yes }}$ of $L$ and rejects a superset $L_{\text {no }}$ of $K \backslash L$, where $L_{\text {yes }}$ and $L_{\text {no }}$ are disjoint.

Lemma 5.1. There is a deterministic protocol $D$ which accepts at least $|L| /|Q|^{2}$ strings from $L$ and rejects all strings from $K \backslash L$. No string is left undecided and no string is accepted as well as rejected. Moreover, at most $|Q|^{O\left(t^{2} \cdot \log _{2}|Q|\right)}$ messages are exchanged.

Proof. We begin by fixing a critical pair $\left(p_{0}, p_{1}\right)$ such that at least $|L| /|Q|^{2}$ strings are accepted in the detection problem of $\left(p_{0}, p_{1}\right)$. Observe that such a critical pair exists as a consequence of Lemma 3.4 (b), since each string in $L$ is accepted in the detection problem of at least one critical pair and there are at most $|Q|^{2}$ critical pairs.

Let $L_{\text {yes }}$ be the subset of $L$ which is accepted in the detection problem of $\left(p_{0}, p_{1}\right)$ and let $L_{\mathrm{no}}$ be the superset of $K \backslash L$ of rejected strings. According to Lemma 4.1 there is a nondeterministic protocol $P$ which solves the detection problem of $\left(p_{0}, p_{1}\right)$ with at most $|Q|^{O(t)}$ messages. Thus there are conventional nondeterministic protocols $P_{\text {yes }}$ for $L_{\text {yes }}$ and $P_{\text {no }}$ for $L_{\text {no }}$ which exchange at most $|Q|^{O(t)}$ messages each. 
To obtain a deterministic protocol $D$ from $P_{\text {yes }}$ and $P_{\text {no }}$ we utilize that deterministic protocols with $M^{O\left(\log _{2} M\right)}$ messages can be built from nondeterministic protocols, provided the protocols recognize a language and its complement by exchanging at most $M$ messages [1]. Our situation however is more complicated, since $L_{\text {yes }}$ is only a subset of the complement of $L_{\text {no. }}$. We employ the construction in [5] with the following modifications. Define the communication matrix $C$ of $\left(P_{\text {yes }}, P_{\text {no }}\right)$ by setting

$$
C\left[x_{1} \cdots x_{t}, y_{1} \cdots y_{t}\right]= \begin{cases}1 & x_{1} y_{1} \cdots x_{t} y_{t} \in L_{\mathrm{yes}}, \\ 0 & x_{1} y_{1} \cdots x_{t} y_{t} \in L_{\mathrm{no}} \\ \text { undecided } & \text { otherwise }\end{cases}
$$

Each message $m$ corresponds to a submatrix $M$ of $C$ defined by the collection of rows for which the message is sent and the collection of columns for which it is accepted. Now let $M$ be a submatrix of the communication matrix $C$. We define $\Delta_{\text {yes }}(M)$ to be the maximal size of a submatrix $T$ of $M$, where $T$, after a suitable permutation of rows and columns of $M$, is a lower triangular matrix with ones on the diagonal and zeroes above the diagonal. (Observe that $T$ may contain undecided entries, but these entries have to appear below the diagonal.) Since $L_{\text {yes }}$ is accepted by the nondeterministic protocol $P_{\text {yes }}$ and since no two diagonal entries can be accepted by the same message, we obtain that $\Delta_{\text {yes }}(C)$ is bounded by the number of messages of $P_{\text {yes }}$ and hence $\Delta_{\text {yes }}(C) \leq|Q|^{O(t)}$ follows.

We first try to reject the given input by deterministically selecting a sequence $m_{i}$ of messages from the protocol $P_{\text {no }}$. As for the conventional transformation to deterministic protocols, the triangular message complexity will be halved in each step and in particular $\Delta_{\text {yes }}\left(M_{1} \cap \cdots \cap M_{i}\right) \leq \Delta_{\text {yes }}(C) / 2^{i}$ follows. We proceed as in the conventional transformation and stop the communication prematurely, if the output "no" can be excluded and output "yes". Otherwise, after at most $\log _{2} \Delta_{\text {yes }}(C)$ rounds, we obtain $\Delta_{\text {yes }}\left(M_{1} \cap \cdots \cap M_{i}\right) \leq 1$. As a consequence, the submatrix $M_{1} \cap \cdots \cap M_{i}$ has no triangular submatrix of size two or larger. In particular, the submatrix $M$ of $M_{1} \cdots M_{i}$ spanned by all rows and columns of $M_{i}^{*}$ with a one, contains all ones of $M_{1} \cdots M_{i}$, no zeroes and possibly undecided entries. If the joint input belongs to $M$, then we stop and accept, resp. stop and reject otherwise. In each round only messages of $P_{\text {no }}$ and hence at most $|Q|^{O(t)}$ messages are exchanged. Thus overall at most $\left[|Q|^{O(t)}\right]^{\log _{2} \Delta_{\text {yes }}(C)}=|Q|^{O\left(t^{2} \cdot \log _{2}|Q|\right)}$ messages are generated.

Remember that $L=\left(L_{r}\right)^{t}$, where $L_{r}$ is the language of non-disjointness for $r$-element subsets of $\left\{1, \ldots, r^{32}\right\}$. Let $D$ be a deterministic protocol which accepts only strings in $L$. Also let $\alpha$ be a sufficiently small positive constant. We apply Fact 1.1 and obtain that $D$ accepts at most $|L| / 2^{\alpha \cdot t}$ strings from $L$, provided at most $2^{\alpha \cdot r \cdot t}$ messages are exchanged.

Now, if an NFA $N$ with sublinear ambiguity recognizes $\exists_{1}(L)$, then we apply Lemma 5.1 to obtain a deterministic protocol which exchanges at most $|Q|^{O\left(t^{2} \cdot \log _{2}|Q|\right)}$ messages, accepts at least $\left|L_{r}\right| /|Q|^{2}$ strings and accepts only strings from $L$. Thus, if $|Q|^{O\left(t^{2} \cdot \log _{2}|Q|\right)} \leq 2^{\alpha \cdot r \cdot t}$ for a sufficiently small positive constant $\alpha$, then at most $|L| / 2^{\alpha \cdot t}$ inputs from $L$ are accepted. But the nondeterministic protocol accepts at least $|L| /|Q|^{2}$ strings from $L$ and hence

$$
|Q|=2^{\Omega(t)}
$$

follows. We set $t=r^{1 / 3}$. Let $\beta$ be a sufficiently small positive constant. Now either $|Q| \geq 2^{\beta \cdot \sqrt{r / t}}$ and we are done, since then $|Q|=2^{\Omega\left(r^{1 / 3}\right)}$ or $|Q|<2^{\beta \cdot \sqrt{r / t}}$ holds. In the latter case

$$
|Q|^{t^{2} \cdot \log _{2}|Q|}<2^{(\beta \cdot \sqrt{r / t}) \cdot t^{2} \cdot(\beta \cdot \sqrt{r / t})}=2^{\beta^{2} \cdot t \cdot r}
$$


and the upper bound on the number of messages in Fact 1.1 is met, provided $\beta$ is sufficiently small. But then $|Q|=2^{\Omega(t)}$ follows from (5.1) and hence $|Q| \geq 2^{\gamma \cdot t}$ holds for some positive constant $\gamma$. We obtain $2^{\gamma \cdot t} \leq|Q|<2^{\beta \cdot \sqrt{r / t}}$ and hence $2^{\gamma \cdot t}<2^{\beta \cdot \sqrt{r / t}}=2^{\beta \cdot t}$, since $t=r^{1 / 3}$. We get a contradiction if $\beta$ is chosen sufficiently small and we have shown

Lemma 5.2. Let $N$ be an NFA with sublinear ambiguity recognizing $\exists_{1}(L)$. Then $N$ has at least $2^{\Omega\left(r^{1 / 3}\right)}$ states.

\section{A Hierarchy for Polynomial Ambiguity}

Let $k \geq 1$ be arbitrary and let $N$ be an NFA for $\exists_{k}(L)$. We again follow the strategy for $k=1$, however the transition from NFA's to communication protocols is now more involved. For $k>1$ we have to work with vectors $\left(p_{0}, p_{1}, \ldots, p_{2(k-1)}, p_{2(k-1)+1}\right)$ of states and besides reachabilty for $p_{0}$ and acceptance by $p_{2(k-1)+1}$ we also have to guarantee that computation paths exist between $p_{2 i}$ and $p_{2 i+1}$. This last requirement requires some further work.

Definition 6.1. Let $\left(\xi_{0}, \xi_{1}\right) \in \exists_{=0}(L) \times \exists_{=0}(L)$ be arbitrary. We say that the vector $\left(p_{0}, p_{1}, \ldots, p_{2(k-1)}, p_{2(k-1)+1}\right)$ is critical for $\left(\xi_{0}, \xi_{1}\right)$ iff

(1) all strings in $\exists_{=0}(L) \cdot \xi_{0}$ reach $p_{0}$ and all strings in $\xi_{1} \cdot \exists_{=0}(L)$ are accepted by $p_{2(k-1)+1}$

(2) and for all strings $u \in \exists_{=0}(L)$ and for all $i(0 \leq i<k-1)$ there is a string $v$ such that a computation for $\xi_{1} u v$ starts in $p_{2 i+1}$ and ends in $p_{2(i+1)}$.

We construct $\xi_{0}$ as in Lemma 3.2 and hence for any state $p$ of the NFA $N$ either all strings in $\exists_{=0}(L) \cdot \xi_{0}$ reach $p$ or no such string reaches $p$. To construct $\xi_{1}$ we first run the procedure of Lemma 3.2 and property (1) is satisfied. Then we process all pairs $(p, q)$ of states of $N$ in some arbitrary order. If for all strings $u \in \exists_{=0}(L)$ there is a string $v \in \exists_{=0}(L)$ such that $\xi_{1} u v$ has a computation beginning in $p$ and ending in $q$, then we say that the pair $(p, q)$ is "alive" and $\xi_{1}$ is left unchanged. Otherwise there is a string $u \in \exists_{=0}(L)$ such that no computation for a string in $\xi_{1} \cdot u \cdot \exists_{=0}(L)$ has a computation beginning in $p$ and ending in $q$. We replace $\xi_{1}$ by $\xi_{1} u$. The pair $(p, q)$ is now "dead", since no string in $\xi_{1} \cdot \exists_{=0}(L)$ has a computation beginning in $p$ and ending in $q$. Also observe that processed pairs do not change their status, i.e., remain dead, resp. stay alive after updating $\xi_{1}$. We have generalized Lemma 3.2.

Lemma 6.2. Let $N$ be an NFA for $\exists_{k}(L)$. Then there are strings $\xi_{0}, \xi_{1} \in \exists_{=0}(L)$ such that

$$
\begin{aligned}
& \left\{z \in L \mid p_{2 i+1} \stackrel{z}{\rightarrow} p_{2(i+1)} \text { for all } 0 \leq i<k-1\right\}=L \text {. } \\
& \left(p_{0}, \ldots, p_{2 k-1}\right) \text { is critical for }\left(\xi_{0}, \xi_{1}\right)
\end{aligned}
$$

Proof. The argument is analogous to the proof of Lemma 3.2. This time we have to observe that accepting computations for strings in $\xi_{0} \cdot\left(L \cdot \xi_{1}\right)^{k}$ have to traverse critical vectors.

For $k=1$ Lemma 3.3 establishes that a string $S(z)$ "lives" in a launching cycle for $p_{0}$ and a storage cycle for $p_{1}$. Its generalization requires more work. Let $\vec{p}=\left(p_{0}, \ldots, p_{2 k-1}\right)$ be a critical vector and let $z \in K \backslash L$ be an arbitrary string. We construct a string $u \in \exists_{=0}(L)$ for $z$ so that some string with period $u z$ can be launched by $p_{0}$, stored and launched in between $p_{2 i+1}$ and $p_{2(i+1)}$ and finally stored by $p_{2(k-1)+1}$. In particular, we say that a string $u \in \exists_{=0}(L)$ is appropriate for $z$ if the following properties are satisfied: 
(1) $(z u)^{|Q|}$ reaches $p_{0}$.

(2) For every $i, 0 \leq i<k-1$, there is a string $s_{i}$ and computations $p_{2 i+1} \stackrel{s_{i}}{\rightarrow} p_{2(i+1)}$. Moreover, $s_{i}$ starts with a suffix of $u z$ containing $\xi_{1}$ as prefix, followed by $(u z)^{|Q|}$ and completed by a prefix of $u$.

(3) State $p_{2(k-1)+1}$ accepts any string $s_{k-1}$ which consists of a suffix of $u z$ containing $\xi_{1}$ as prefix, followed by $(u z)^{|Q|}$.

(4) The string $s=(z u)^{|Q|} z s_{0} z \cdots z s_{k-2} z s_{k-1}$ has periods $z u$ and $u z$ respectively.

Now assume that $u$ is appropriate for $z$. We show that the string $S(z)=s$ "lives" in appropriate cycles for each $p_{i}$. First observe that $S(z)$ has period $z u$ and hence also period $u z$. The proof of Lemma 3.3 shows that a launching cycle $r \stackrel{(z u)^{a}}{\rightarrow} r \stackrel{(z u)^{a_{1}}}{\rightarrow} p_{0}$ is established, once $(z u)^{|Q|}$ reaches $p_{0}$. Also, intermediate cycles in between $p_{2 i+1}$ and $p_{2(i+1)}$ exist, since $s_{i}$ has substring $(u z)^{|Q|}$, and a final storage cycle following $p_{2(k-1)+1}$ exists, since $p_{2(k-1)+1}$ accepts a string with suffix $(u z)^{|Q|}$.

Lemma 6.3. For every string $z \in K \backslash L$ there is an appropriate string $u \in \exists_{=0}(L)$ for $z$.

Proof. Let $q_{l}$ be some arbitrary ordering of the states of $N$. Each pair $\left(p_{2 i+1}, p_{2(i+1)}\right)$ influences the construction of $u$. Assume for the moment that strings $\xi_{i, l}$ are already defined. We set

$$
u_{i, j}=\xi_{1} \cdot \Pi_{l \leq j,\left(q_{l}, p_{2(i+1)}\right)} \text { is alive } z \xi_{1} \xi_{i, l}
$$

for all $j(1 \leq j \leq|Q|)$. Observe that $u_{i, j}=u_{i, j-1} \cdot\left(z \xi_{1} \xi_{i, j}\right)$, if $\left(q_{j}, p_{2(i+1)}\right)$ is alive, and that $\xi_{1}$ is a prefix of $u_{i, j}$. Choose the strings $\xi_{i, l} \in \exists_{=0}(L)$ so that there is a computation for $u_{i, j}$ from $q_{j}$ to $p_{2(i+1)}$. Such strings $\xi_{i, l}$ exist with property (2) of a critical vector, since $\xi_{1}$ is a prefix of $u_{i, j}$ and $\left(q_{j}, p_{2(i+1)}\right)$ is alive. Finally set

$$
u_{i}=u_{i,|Q|} \cdot z \xi_{1} \text { and } u=u_{0} \cdots u_{k-2} \cdot \xi_{0} .
$$

We show that $u$ is appropriate for $z$ by first verifying property (1). The string $u$ has suffix $\xi_{0}$ and hence, by property (1) of a critical vector, $(z u)^{|Q|}$ reaches $p_{0}$, the first component of the critical vector $\vec{p}$.

Observe that each $u_{i, j}$ has prefix $\xi_{1}$ and hence $u_{i}$ and $u$ have $\xi_{1}$ as prefix. We start the verification of properties (2) and (3) by defining $s_{0}$ and constructing a computation $p_{1} \stackrel{s_{0}}{\rightarrow} p_{2}$. Since $\xi_{1}$ is a prefix of $u$, there is a computation for $(u z)^{|Q|}$ which leads from $p_{1}$ to a state $q_{j}$ such that the pair $\left(q_{j}, p_{2}\right)$ is alive. But then, by definition of $u_{0, j}$, there is a computation for $(u z)^{|Q|} \cdot u_{0, j}$ which starts in $p_{1}$, reaches $q_{j}$ after reading $(u z)^{|Q|}$ and ends in $p_{2}$ after reading $u_{0, j}$. We set $s_{0}=(u z)^{|Q|} \cdot u_{0, j}$. By construction, $u_{0, j}$ is a prefix of $u_{0}$ which itself is a prefix of $u$. Thus there is a string $v_{0, j}$ with $u=u_{0, j} \cdot z \cdot v_{0, j}$ and $v_{0, j}$ has prefix $\xi_{1}$.

We now construct a string $s_{1}$ and a computation $p_{3} \stackrel{s_{1}}{\rightarrow} p_{4}$ as follows. Since $v_{0, j}$ has prefix $\xi_{1}$ there is a computation for $v_{0, j} \cdot z \cdot(u z)^{|Q|} \cdot u_{0}$ which reaches a state $q_{k}$ when starting in state $p_{3}$. Since the pair $\left(q_{k}, p_{4}\right)$ is alive, we obtain the computation

$$
p_{3} \stackrel{v_{0, j} z(u z)^{|Q|} u_{0}}{\rightarrow} q_{k} \stackrel{u_{1, k}}{\rightarrow} p_{4}
$$

and set $s_{1}=v_{0, j} z(u z)^{|Q|} u_{0} u_{1, k}$. The construction of $s_{i}$ and verifying a computation $p_{2 i+1} \stackrel{s_{i}}{\rightarrow}$ $p_{2(i+1)}$ for arbitrary $i<k-1$ proceeds in a completely analogous fashion. Finally, again by property (1) of a critical vector, state $p_{2(k-1)+1}$ accepts any string $s_{k-1}$ consisting of a suffix of $u z$ followed by $(z u)^{|Q|}$, since the suffix of $u z$ has prefix $\xi_{1}$. 
To complete the argument observe that by construction $s=(z u)^{|Q|} z s_{0} z \cdots z s_{k-2} z s_{k-1}$ has periods $u z$ and $z u$ respectively.

The remainder of the argument proceeds completely analogous to the case of $k=1$. Lemma 3.4 shows that an NFA with sublinear ambiguity solves the detection problem for $k=1$. To introduce its generalization we firstly introduce the detection problem for $k>1$ : $z$ has to be rejected iff there is a string $z^{\prime} \in K \backslash L$ such that $z$, acting as an impostor of $z^{\prime}$, can be placed in matching positions within the $k+1$ individual $u z^{\prime}$-cycles of $N$. Lemma 3.4 was a direct consequence of Lemma 3.3 in the case of $k=1$. In the same manner we can now show that an NFA with ambiguity $o\left(n^{k}\right)$ solves the detection problem for general $k$ as a direct consequence of Lemma 6.3.

Let $N$ be an NFA with ambiguity $o\left(n^{k}\right)$ for $\exists_{k}(L)$. As in Lemma 4.1 we simulate $N$ to obtain a nondeterministic protocol $P$ solving the detection problem with $|Q|^{O(k t)}$ messages; the exponent grows by the factor $k$, since $k+1$ instead of two computations of $N$ on input $z$ have to be simulated. We transform $P$ into a deterministic protocol $D$ with $|Q|^{O\left((k t)^{2} \log |Q|\right)}$ messages as in Lemma 5.1. To complete the proof of Theorem 1.2, we replace $r$ by $r / k^{2}$ in the proof of Lemma 5.2 (to compensate for the increase in the number of messages of $D$ from $|Q|^{O\left(t^{2} \log |Q|\right)}$ to $\left.|Q|^{O\left(k^{2} t^{2} \log |Q|\right)}\right)$ and obtain

Lemma 6.4. Let $N$ be an NFA with ambiguity o $\left(n^{k}\right)$ recognizing $\exists_{k}(L)$. Then $N$ has at least $2^{\Omega\left(\left(r / k^{2}\right)^{1 / 3}\right)}$ states.

\section{References}

[1] Aho, A.V., Ullman, J.D. and Yannakakis, M., On notions of information transfer in VLSI circuits, Proc. of the 15th Annual STOC, pp. 133-139, 1983.

[2] Hromkovič, J., Karhumäki, J., Klauck, H., and Schnitger, G., Communication complexity method for measuring nondeterminism in finite automata, Inf. Comput. 172, pp. 202-217, 2002.

[3] Hromkovič, J., and Schnitger, G., Nondeterministic Communication with a Limited Number of Advice Bits, SIAM J. Comput. 33(1), pp. 43-68, 2003.

[4] Leung, H., Separating exponentially ambiguous finite automata from polynomially ambiguous finite automata, SIAM. J. Comput. 27, pp. 1073-1082, 1998.

[5] Lovasz, L., Communication complexity: a survey, in "Paths, Flows and VLSI Layout", Korte, Lovasz, Prömel, Schrijver eds., Springer Verlag, pp. 235-266, 1990.

[6] Ravikumar, B., and Ibarra, O., Relating the type of ambiguity of finite automata to the succinctness of their representation, SIAM J. Comput. 19, pp. 1263-1282, 1989.

[7] R.E. Stearns and H.B. Hunt III, On the equivalence and containment problems for unambiguous regular expressions, regular grammars and finite automata, SIAM J. Comput. 14(3), pp. 598-611, 1985.

[8] A. Weber and H. Seidl, On the degree of ambiguity of finite automata, Theor. Comput. Sci. 88 (2), pp. 325-349, 1991. 